\title{
9 \\ Objective Chance: Lonergan and Peirce on Scientific Generalization
}

IN MANY RESPECTS the views of Bernard Lonergan and Charles Peirce concerning world process are strikingly similar. Thus, both outline an evolutionary cosmology ${ }^{1}$ that pays attention to both the law-like and the chance elements required to think of the universe as developing. Both reject the notion that the universe is mechanistically determined even if it is ordered. Both look upon "chance" as an objective component of the universe, not merely as a cloak for our ignorance. The remarkable convergence of ideas of two thinkers separated by almost a century not only illuminates their place in intellectual history but, more important, adds an extrinsic confirmation of a cosmological view that takes motion and change seriously.

As might be expected, random differences play an important role in both Lonergan's and Peirce's account of physical laws. The insight into the significance of such differences amounts to this: the universe is in a process of growth and development from a state of lesser to greater complexity. Lonergan defines a situation as random "if it is 'any [situation] whatever, provided specific conditions of intelligibility are not filled."'2 The "specified conditions of intelligibility" are those provided by a systematic understanding of the process. To the extent to which a situation fails to fall under such a set of conditions, it is non-systematic or random.

An earlier version of this chapter appeared in Method: Journal of Lonergan Studies, 12 (1994), 91-107. 


\section{LONERGAN}

Let us consider first Lonergan's account of physical laws, and let us begin with his distinction between systematic and non-systematic processes. It is not so simple and clear-cut as it might at first seem. First of all, it is at least unusual to talk of a process that is nonsystematic. It would seem that for a process to be a process at all it must be ordered in some way and thus be "systematic" in some commonsense meaning of the term. Of course, the point is that Lonergan uses the terms "systematic" and "non-systematic" in a technical, not a commonsense, meaning. In short, he uses them to indicate a definite type of order and its absence, as we shall see shortly. Even when we realize that Lonergan uses these terms technically, they remain difficult to grasp, because in experience processes always show both a systematic and a non-systematic aspect. They can be distinguished in understanding, but they cannot be separated in experience. They are, then, for Lonergan, complementary; but we must not allow their complementarity to obscure their real distinction lest we lose the significance of randomness for understanding our world.

A process is said to be systematic if it can be grasped in its entirety in a single insight or in a single set of unified insights. ${ }^{3}$ Classical physics, for example, seeks to grasp such process precisely as systematic by looking for an answer to the question "What kind of process is this?" or, perhaps better, "What is the nature of this phenomenon?" It does so precisely by correlating measurable relevant variables (time, speed, temperature, pressure, or whatever) into differential equations. These equations are used to predict future events of the appropriate kind.

Systematic process then is commanded, as it were, by a single idea which allows us to know a priori and in principle (through differential equations expressing laws) all the events/situations that make up the process. Consequently, any event or situation in the process can be deduced from any other by virtue of the idée maîtresse. In a word, these physical processes are regarded as being subsumed under a covering law and so are ordered deductively. ${ }^{4}$ Such a grasp of events/situations possesses a tremendous predictive power. It enables us to organize vast amounts of empiri- 
cal data and to make predictions which are regularly fulfilled. ${ }^{5}$ The insight into physical processes as systematic is that of so-called "classical" physics.

A process is said to be non-systematic if it has not been (and indeed ultimately cannot be) grasped in a single insight or set of unified insights. In such a case there is no one idea that governs the whole, and so one cannot deduce any event/situation from any other. (This does not mean that one cannot infer an event/situation from some other[s]; not all inference is deductive.) At best, predictions are based on probabilities calculated statistically. Indeed, Lonergan calls this model of science "statistical" physics, and he sees it as complementary to the classical model.

These models of science, classical and statistical, are heuristics. An heuristic is an intelligent anticipation of intelligibility of a certain kind. Thus, classical heuristic anticipates the systematic and abstract (the physical laws) on which the concrete observations converge. Statistical heuristic anticipates the systematic and abstract which sets a boundary (the ideal frequency) from which the concrete cannot systematically diverge (allows random divergence).

The statistical model deals with events as "coincidental aggregates." Events are assumed to have no relations to one another other than mere juxtaposition in space. Classical physics deals with events as intelligible wholes causally related. The laws that it formulates, however, always have added (explicitly or implicitly) to its generalizations the phrase "other things being equal." Hence, classical physics expects that there will be no differences between predicted and observed results. If such differences do appear, they are put down either to "observational error" or to arbitrary isolation of part of the physical universe, and so the observed results are expected to converge on the predicted as the observational errors or arbitrary isolation is overcome. To be sure, classical physics used statistical analysis when dealing with very, very large numbers (for example, the number of gas molecules in a container), but this was considered to be an unfortunate second-best and so to be a "cloak of our ignorance" of the actual behavior of such large populations. In principle, then, if one were to adopt the classical model as uniquely correct, statistical methods would be expected to be gradually eliminated.

Statistical physics, on the other hand, renders an account of 
discrepancies between predicted and observed results, thus giving them a kind of intelligibility by distinguishing deviations which are merely random from deviations which are themselves systematic. It sets limits (ideal frequencies) from which observed data cannot diverge systematically. ${ }^{6}$ Non-systematic processes, then, manifest a certain intelligibility and thus a certain order, but an order and intelligibility different from that of systematic processes. Hence, statistical investigation, which deals with non-systematic process, anticipates a different intelligibility than does classical. The order studied by statistical investigation is that of actual relative frequencies of events as diverging from an ideal frequency only at random. In effect, it counts the number of times outcomes of a certain type actually occur. Thus, for example, the ratio of heads or of tails in tossing a fair coin turns out to be one half since the actual ratio of each for any series of tosses oscillates at random about the value $1 / 2 .{ }^{7}$ Statistical techniques can then reveal a certain order in a run of events which from a classical point of view has no immanent intelligibility. The mind-set (heuristic anticipation) fostered by statistics is to expect that there will be differences which do not make a difference provided they are merely random. The classical mindset, however, expects every difference to make a difference and puts down divergences from predicted values to observational error or to the arbitrary isolation of a physical system.

Perhaps an example will make the point clearer. According to classical kinetic theory, the interrelations of pressure, volume, and temperature of a gas (Boyle's and Charles's laws) are accounted for by supposing that the gas is composed of a very large number of molecules moving at random inside the container. Any molecule has as much chance as any other of hitting the container's wall. It was noted that the predicted values calculated for pressure or volume or temperature using statistical methods were only approximated by the actual values when observed. It was thought that, because every difference must make a difference, if the laws governing the relations between pressure, volume, and temperature were correct, the discrepancies must be due to faulty measurement and/or observational techniques. The expectation then was: improve the accuracy of measurement and observation and you will reduce the discrepancies between predicted and observed values. It turned out, however, that just the opposite resulted; the more 
accurate the observations the wider the divergence. Something, then, must have been wrong with the theory; it must be missing something. This realization led scientists completely to rethink Newtonian (classical) mechanics on which kinetic theory was built. The outcome, of course, was the elaboration of quantum mechanics.

It turns out, then, that statistical techniques do indeed give to runs of events a certain intelligibility indirectly and from the outside as it were-through the mathematics of probability, not through the unity of cause and of nature. In the case of deviations from the ideal frequency of any run of events, since they are all merely random they do not have even the intelligibility of nonsystematic process. They are in fact the surd element of concreteness and finiteness. ${ }^{8}$ In the real order, then, one finds no processes that are entirely systematic and no processes that are entirely nonsystematic. Every real, observable world process exhibits aspects of each.

Both classical and statistical physics seek intelligibility in actual physical processes. Each, then, formulates "laws," and in each case observed values and predicted values differ; in the case of classical physics, those differences are unexpected and unwanted (merely "observational error"); in the case of statistical physics, they are expected and are permitted if and only if the differences are random. In both heuristics there is an inverse insight into the differences between observed and predicted results. In the case of classical heuristic, they are allowed as long as they are only the result of observational error that can be corrected by more careful and accurate measurement; in the case of statistical heuristic, they are allowed only when deviating from the ideal frequency at random.

Thus, the negative unintelligibility of random differences which make no difference to the generalization (the ideal frequency) is simply a recognition of the abstract character of our explanatory generalization. The positive unintelligibility of differences too large to be set down to mere observational error and too systematic to be ignored is recognition that our explanatory generalization has missed some relevant variable in the process and thus forces revision of the differential equation. Lonergan puts the matter like this: "when differences are not random (too large to be merely 
random) further inquiry is in order; but when differences are random, not only is no inquiry attempted but also the very attempt would be pronounced silly."9

Because of the complementarity of classical and statistical heuristics implied in the foregoing, they are not to be regarded as competing views. On the contrary, they are to be regarded as contributing positively to each other. Thus, to return to the example of kinetic theory cited above: the anticipation that the laws of gases as worked out in Newtonian mechanics would be perfectly confirmed by the coincidence of predicted and observed results was disappointed by further research, so that the very laws of mechanics had to be reformulated in such a way as to incorporate statistical method into the essence of the theory and not leave those statistical outcomes as simply a cover for ignorance.

Since these heuristics are truly complementary, it is plausible to assume $a$ priori that they are irreducible in the sense that the greater development of one heuristic will not result in the elimination of the other. Suppose that classical physics succeeds in developing ever more inclusive frameworks in which to organize its data into more and more inclusive laws. Among other things this would mean that what was omitted by one framework was included in the next. Still, in the more inclusive framework there would be other items which escape the new laws. In every case these items which elude inclusion under classical law are considered in terms of statistical laws and are judged to be either random or systematic deviations. If the deviations are statistically significant, further investigation is in order. If they are merely random, no further research is required. In that case we are simply reflecting that the concrete, real situation reflects an empirical residue which can never be captured in laws whether classical or statistical. In a word, no matter how inclusive a framework is developed, in the concrete random differences will never be eliminated. Why is this so? What is the significance of differences which do not make a difference to any physical theory in question?

The classical heuristic if taken alone would have deduction as its model. Gödel proved that no non-trivial deductive system can be shown within the system to be both consistent and deductively complete. Deductive completeness means that every true statement appear as part of the system either as an axiom or as a theo- 
rem. Consistency, of course, means that the system is not selfcontradictory. Clearly, all logicians want a deductive system to be at least consistent. But Gödel showed that no consistent, non-trivial deductive system can contain all true statements. There will always be at least one statement known to be true which is neither an axiom nor a theorem of the system. If one tries to fix up the axioms so that the truth previously not contained in the system is now so contained, another, different, statement will be found which, while known to be true, is neither an axiom nor a theorem. These "undecidables" are to the axiomatic system as the empirical residue is to classical physics. The strange, even disconcerting phenomenon is due, I think, to the abstract nature of our expressions of the world's intelligibility in general principles and laws. The reality of the concrete always involves more than the abstract can express.

The question whether world processes are systematic or not is an empirical question. It seems to have been settled that they in fact manifest aspects of each. Hence, insofar as there were a nonsystematic aspect to actual world processes, the randomness they manifest would not be just "a cloak for our ignorance," but rather a property of what exists.

Lonergan points out that even if we accept that classical and statistical heuristics, correctly understood, are complementary, we still need an explanatory idea which accounts for the characteristics of each of those anticipatory schemes. As he remarks, the laws formulated under the classical heuristic (for example, Newtonian mechanics) not only do not give any insight into numbers, distribution, intervals of time, selectivity, and other elements that enter into statistical analyses, but rather abstract from all particulars and concrete conditions under which those classical laws actually function. ${ }^{10}$ On the other hand, laws formulated under the statistical heuristic (for example, quantum mechanics) give no account of why there are "so many kinds of events or why each kind has the frequency attributed to it." 11 They merely provide in various cases an ideal frequency of the occurrence of the events. Lonergan concludes:

To reach explanation on this level, it is necessary to effect the concrete synthesis of classical laws into a conditioned series of schemes of recurrence, to establish that such schemes, as combinations of 
events, acquire first a probability of emergency and then a probability of survival through the realization of the conditioned series, and finally to grasp that, if such a series of schemes is being realized in accord with probabilities, then there is available a general principle that promises answers to questions about the reason for numbers and distributions, concentrations and time intervals, selectivity and uncertain stability, development and breakdowns. To work out the answers pertains to the natural sciences. To grasp that emergent probability is an explanatory idea, is to know what is meant when our objective was characterized as a generic, relatively invariant, and incomplete account of the immanent intelligibility, the order, the design of the universe of our experience. ${ }^{12}$

It is further evident that Lonergan is satisfied that the world process is, at least in some significant cases, non-systematic. In the section of Insight dealing with "emergent probability,"13 he spells out in some detail what is to be expected from an empirical examination of the actual world if indeed both classical and statistical laws were supposed to be true of that world. We would then expect that world to exhibit both regularities and novelties, both lawful and chance events - in short, be a world of continuity and differentiation. We have only to go and look to judge whether such an heuristic anticipation is justified by empirical testing.

\section{PeIRCE}

Consider now what Charles Peirce has to say of the same subject. $\mathrm{He}$ is writing just before the turn of the century and so before Einstein, Heisenberg, and Planck. His notion of physics has been formed largely in the Newtonian, and so classical, model in which emphasis has been laid on events converging on predicted outcomes and in which divergence of actual observations from the norm were considered to be due to observational error of one kind or another. And yet in a paper published in 1893 in The Monist, he remarked:

The Origin of Species was published toward the end of the year 1859. The preceding years since 1846 had been one of the most productive seasons-or if extended so as to cover the great book we are considering, the most productive period of equal length in the 
entire history of science from its beginning until now. The idea that chance begets order, which is one of the cornerstones of modern physics . . . was at that time put into its clearest light. (6.297)

Peirce was convinced that Darwin's evolutionism and any mechanistic interpretation given to science and its laws were incompatible. In Peirce's opinion the use of statistical methods in science did not give any aid or comfort to "mechanical philosophy" as some, among them Peirce's friend Chauncey Wright (see, 5.64), supposed. To the contrary, Peirce regarded statistical methods as sounding the death knell for such views.

For Peirce the "mechanical philosopher" is "whoever holds that every act of the will as well as every idea of the mind is under the rigid governance of a necessity coordinated with that of the physical world" (6.38). Such a philosopher "will logically be carried to the proposition that minds are part of the physical world in such a sense that the laws of mechanics determine anything that happens according to immutable attractions and repulsions" (6.38). Peirce called this "the usual and most logical form of necessitarianism." We recognize in this "mechanical philosophy" the mind-set Lonergan spoke of as arising from the mistaken assumption that the classical model of physics is the uniquely correct one. It is against this view of things that Peirce, like Lonergan many years later, mustered all his considerable logical, scientific, and philosophical resources.

What, Peirce asks, are the reasons for holding such a view? Three sorts of arguments are usually proposed: (l) absolute determinism is a postulate of scientific reasoning; (2) absolute determinism is supported by observational evidence; and (3) various a priori arguments support it (6.39-65). The first class of arguments is based on a faulty understanding of scientific method; the second is simply gratuitously asserted; and the third does not exhaust all possible alternative hypotheses, in particular, the hypothesis that chance is in some sense real and not merely a function of our ignorance.

Let us consider Peirce's criticisms more closely. To think that absolute determinism is a postulate of scientific reasoning is to assume erroneously that induction is nothing but a special case of deduction. Peirce, of course, has in mind the sort of analysis of 
induction which grounds its effectiveness in the uniformity of nature. But he points out that in fact all inductive inference is based on the principle of sampling. He gives this example: from samples of a shipment of wheat we find that four-fifths of the samples contain quality A wheat; hence, we conclude "experientially and provisionally" (Peirce's words) that about four-fifths of all the grain in the shipment is of the same quality. Such an argument is based solely on what we have experienced of the wheat in the past and what we can experience of it in the future. Because that sort of reasoning is conditioned by experience (both actual and possible), its conclusion concerning the true proportion of quality A wheat in the shipment is only provisional. At any given moment, therefore, the inferred ratio is only an approximation of what would be found to be the case if more samples were to be taken. The inferred ratio, then, is really an hypothesis to be verified and/or modified by experience. If the outcomes of further sampling fluctuate irregularly so that no definite value can be assigned to this ratio, the limits within which it fluctuates can be discovered. If, however, further sampling yields definite ratios that change, that fact can be ascertained and the approximation modified accordingly. Thus Peirce remarks:

in short, whatever may be the variations of this ratio in experience, experience indefinitely extended will enable us to detect them, so as to predict rightly, at least, what its ultimate value may be, if it has any ultimate value, or what the ultimate law of succession of values may be, if there be any such law, or that it fluctuates irregularly within certain limits, if it does so ultimately fluctuate. (6.40)

For Peirce, then, the process of sampling is self-corrective and because it is this process which grounds inductive inference, that type of inference needs no postulates whatever, not to mention a postulate of absolute determinism.

As for the claim that there is observational support for absolute determinism, Pierce cannot understand how anyone acquainted with scientific research from the inside could take it seriously. $\mathrm{He}$ points out that any scientist knows that no observation determines the value of a continuous quantity with a probable error of zero. But what about observations of continuous quantities which are discontinuous at one or two limits? For example, take a line seg- 
ment drawn by someone on a sheet of paper. How to determine its length? Since it cannot have a length of less than zero, if no length at all is visible, the observed length is zero. All this means, however, is that the length of the line segment is less than the smallest length visible with the optical power employed. It does not immediately warrant the conclusion that no line segment had been drawn at all. To reach that conclusion legitimately one would have to have recourse to some indirect evidence, for example, that the person who supposedly drew the line in the first place was never anywhere near enough to the paper to do so (6.45-46). Peirce's general point is this: to conclude that some quantity or other is absent from a certain subject matter, there must be some experimental evidence, direct or indirect, to that effect. It is not enough simply to say that we are unable to detect it. Peirce points out further that when we try to verify a law of nature, the more precise our observations, the more certainly will they show irregular departures from the law (6.46).

Finally, consider Peirce's evaluation of the various a priori arguments advanced by the defenders of "mechanical philosophy." The most serious of these, in Peirce's view, is the claim that absolute chance cannot be an explanation of anything. The issue, then, is to determine in what an explanation consists and when one is required. Peirce maintains that regularity, not irregularity, is what par excellence requires an explanation. The reason is that an explanation is required only when questions arise, and questions arise only when there is some thwarted or disappointed expectation. Now, irregularity engenders no expectations whatever as to what is likely to turn up. Hence, it raises no questions and so needs no explanation. Again, purely formal regularities such as those found in mathematical laws (say, of probability) require no explanation either, since they are simply part of the a priori conditions of our knowing randomness at all. The situations that require explanation are: (l) those empirically observed in nature since they are the exception to the preponderance of our experience; (2) breaches in empirically observed regularities since they disappoint our expectations and so raise questions; and (3) failure to discover empirical confirmation of a postulated regularity. In this third case, what needs explanation is not precisely why there is no regularity, but rather why we were led to postulate it in the first place. In Peirce's 
view, then, in a universe such as ours regularity alone needs explanation. Consequently, law cannot be posited as the ultimate explanation of the cosmos. Such a position would lead to this rather curious reasoning: on the one hand, since law and regularity cannot explain irregularity and growing diversity, the latter would have to be set down as inexplicable; on the other hand, if laws cannot have evolved out of irregularity (precisely because of this account they are immutable and ultimate facts), then they have no explanation either. Hence, such a position would block the road to further inquiry and violate what Peirce took to be the most basic tenet of scientific method.

Peirce points out that all the laws of classical mechanics deal with conservative forces, that is, with forces which are reversible and which obey the laws of conservation of energy. He further remarks that in fact most physical phenomena here on earth are non-conservative and so seem to be inexplicable by the laws of classical mechanics: birth, growth, life, friction, heat conduction, combustion, capillarity, diffusion of liquids, etc.

Now, as a general rule, physicists explain those actions which seem to violate the law of conservation of energy through the action of chance. In some cases a uniform distribution can be understood to result from conservative forces acting upon a collection of things whose distribution is fortuitous. This process is known as sifting. The converse is not possible, however. By themselves conservative forces cannot bring about a fortuitous distribution; only another fortuitous distribution in the initial conditions can do that. Peirce gives this example. Suppose a jar to contain hot nitrogen. Add some cold oxygen. At first the nitrogen molecules will be moving with various degrees of force distributed fortuitously. The same will be true of the oxygen molecules. On the average, however, the oxygen molecules will be moving more slowly than the nitrogen. This is not a matter of chance. Furthermore, in the course of time there will be continual fortuitous encounters of the two sorts of molecules causing continual interchange of energy between them, with the result that gradually there will be an approximation to one fortuitous distribution of energy among all the molecules. Peirce observes:

That which happens, happens entirely under the governance of conservative forces; but the character of fortuitous distribution to- 
ward which there is a tendency is entirely due to the various fortuitous distributions existing in the different initial conditions of the motion, with which conservative forces never have anything to do. (6.81)

This is more remarkable, says Peirce, since, although the initial distribution of forces gradually tends to die out, the subsequent fortuitous distributions dependent upon the initial conditions not only hold their ground, but mark their effect wherever the conservative forces act. This is what Peirce means by the "action of chance" (6.81). The uniformity or regularity of a distribution, therefore, can be understood to have come about through the "action of chance" but not conversely. Conservative forces within a mechanical system cannot, by themselves, reverse the sifting process so that a completely fortuitous distribution results from a uniform distribution.

Not all uniform distributions are the result of the sifting of fortuitous distributions, however, and those that are also always involve some sort of regularity in their initial conditions. Peirce gives this example. The density of a gas varies directly with its pressure since more molecules confined to a smaller space will strike the walls of the container per unit time. This is not due to chance alone, however, since the initial conditions suppose that the parts of the molecules are all nearly rectilinear, for otherwise it might turn out that the molecules do not strike the container walls at all even though they are in motion. But such an initial condition is itself a regularity. Hence, regularity in a phenomenon requires some regularity in its initial conditions just as irregularity in a phenomenon requires some irregularity in its initial conditions. Thus regularity and irregularity are ultimate, irreducible, distinct yet inseparable, complementary aspects of all actual world processes. Peirce's hypothetical framework is called synechism (continuity), of which, he says, tychism (objective chance) is but a corollary. These rather exotic terms are perhaps best understood from the Greek roots. "Synechism" is coined from "syn" meaning "along with" or in general "accompanying," and from "echein" meaning "to have" or "to possess." Peirce meant it to stand for regularity of continuity. "Tychism" is coined from "tyche" meaning "chance" with the usual overtone of "good luck" ("dystyche" would mean bad luck). That 
continuity implies randomness as a corollary comes from the mathematics of continuous quantities, that is, their analysis requires the introduction of discreteness and so implies either the notion of limits to which continuous series converge or of infinitesimals of which continuous series are composed. I take it that Peirce looked on this as simply a generalized theory of evolution and that Lonergan would recognize it as the first cousin to his own heuristic hypothesis of emergency probability. ${ }^{14}$

For Peirce, then, the entire universe is in a process of development which can be thought of as hyperbolic:

The state of things in the infinite past is chaos . . . the nothingness of which consists in the total absence of regularity. The state of things in the infinite future is death, the nothingness of which consists in the complete triumph of law and the absence of all spontaneity. (8.317)

These states (infinite past and infinite future) are, however, only theoretical limits which are approached asymptotically. Between them

we have on our side a state of things in which there is some absolute spontaneity [chance] counter to all law, and some degree of conformity to law, which is constantly on the increase owing to the growth of habit. (8.317)

This is for Peirce the law of the universe, the law of habit-taking, the law of mind.

\section{Notes}

1. To the best of my knowledge Lonergan's position as found in Insight: A Study of Human Understanding (New York: Philosophical Library, 1956) was developed quite independently of Peirce's views. Peirce wrote some fifty years before Lonergan and I find no evidence that Lonergan knew Peirce's work until several years after Insight was published.

2. Ibid., p. 51.

3. Ibid., p. 48.

4. Lonergan referred to his theory as "emergent probability" (ibid, pp. 121-28). He characterized it as both generic and explanatory. Peirce referred to his theory as "agapastic evolution," an explanatory account of 
the growth and development of the cosmos which attempts to do justice to the role of the categories in world process by an appeal to the "action of love," that is, mediation between the necessity and the spontaneity of actual world process. I have treated this at some length in Charles $S$. Peirce: On Norms and Ideals (Amherst: University of Massachusetts Press, 1967), pp. 171-90.

Charles Peirce will also hold that classical (Newtonian) physics is deterministic in its view of the physical world because it took deduction as its model of explanation. Gerrit Smith, who was Professor of the Philosophy of Science, Fordham University, and who held his Ph.D. in Physics from Syracuse University, pointed out to me that while Peirce and Lonergan have in mind Newtonian mechanics as "classical physics," the real culprit behind this determinist mentality is more ancient and more profound. It is more ancient in that it goes back to the Pythagoreans and more profound in that it supposes a universe that is static and changeless, and so is capable of being completely expressed mathematically (geometrically, first, and then algebraically). (See the study of his student, Jamila Jauhari, "The Physics of Avicenna," Ph.D. diss., Fordham University, 1987.) Furthermore, the Pythagorean view would not allow anything like an "empirical residue" (Lonergan) or an irradicable "Secondness" (Peirce). Any discrepancy between predicted and observed outcomes would be put down not merely to "observational error" but also to the fact that, whenever an arbitrarily selected portion of the physical universe is treated as an isolated physical system, then, inevitably, some violation of the laws of conservation will appear and hence bring about discrepancies between predicted and observed results.

5. Lonergan, Insight, p. 48.

6. Some may find it helpful to think of "ideal frequency" in more precise, mathematical terms. Again I am in debt to Dr. Smith who brought this to my attention and who offered the following definitions: (a) Cauchy Series: a sequence of numbers is said to be "Cauchy" if and only if for any positive number $\epsilon(\epsilon>0)$, there is a positive integer $N$ such that for members $x_{n}$ and $x_{m}$ of the sequence with both $n$ and $m$ greater than $\mathrm{N},] \mathrm{x}_{\mathrm{n}}-\mathrm{x}_{\mathrm{m}}[<\epsilon$. Hence, the further one proceeds in such a sequence the more closely "bunched up" are the members. (b) Limit " $L$ " of Cauchy Series: A Cauchy series is said to have a limit "L" if and only if for any positive number $\epsilon(\epsilon>0)$, there is a positive number $N$ such that for all $\mathrm{n}>\mathrm{N},]^{\prime \prime} \mathrm{L}$ "- $\mathrm{x}_{\mathrm{n}}\left[>\epsilon\right.$. (c) Ideal Frequency: a sequence $\left(\mathrm{x}_{1} \ldots \mathrm{x}_{\mathrm{n}}\right)$ of statistical outcomes with average $\mathrm{x}$ is said to have an ideal frequency $\mathrm{x}_{\mathrm{id}}$ if for any positive integer $\epsilon(\epsilon>0)$, the probability that $] \mathrm{x}[>] \mathrm{x}_{\mathrm{id}} \pm \epsilon[$ vanishes as $\mathrm{N}$--$>$ infinity.

7. Dr. Smith points out that the difference between the expectations 
for statistical studies in Newtonian mechanics and in quantum mechanics is just what Lonergan says is the difference between classical and statistical heuristic structures. Newtonian mechanics supposes that there are available to the observer other parameters which he simply neglects out of ignorance, laziness, or technological limitations. If these parameters were to be taken into account, the observed data would indeed converge on the predicted results. Quantum mechanics, on the other hand, supposes that there simply are not such other parameters. Lonergan's point is that, because classical and statistical heuristics are complementary, statistical techniques even when applied within classical physics are impossible to dispense with, since their reason for existence is the empirical residue that cannot be eliminated by any abstract laws whether classical or statistical. Statistics is not a mere cloak of our ignorance even in classical investigation. Peirce's agreement with the substance of this view may well have been what led him in fact to anticipate quantum theory. Lonergan's understanding of the physical universe is anti-Pythagorean and prohylemorphic, since "prime matter" is the analogue of the "empirical residue." Peirce is in the same camp by his insistence on "Secondness" as brute.

8. A convincing confirmation of a surd aspect, a brute and factitious element, in nature is the fact that physical constants, like $\mathrm{K}$ (specific gas constant), or $\mathrm{R}$ (universal gas constant), or $\mathrm{C}$ (speed of light), must be empirically measured and calculated. Indeed, a theory may posit that there is such and such a constant, but the theory cannot assign it a numerical value. That value simply is whatever it turns out to be.

9. Insight, pp. 54-55.

10. Ibid., p. 124.

11. Ibid.

12. Ibid.

13. Pp. $115-28$.

14. Here are, according to Lonergan, some of the attributes the universe would in fact manifest if emergent probability were true: (l) there is a succession of world situations each one of which comes about, survives, and changes according to a schedule of probabilities; (2) world process is open, that is, not determined; (3) world process is increasingly systematic; (4) world process admits enormous differentiation; (5) world process admits of breakdowns; (6) world process includes blind alleys; (7) the later a scheme is in the conditioned series of schemes, the narrower its distribution in the cosmos; (8) the narrower the basis for the emergency of the later schemes of emergence, the longer the time interval; (9) the greater the probability of blind alleys and breakdowns, the greater must be the initial number if the whole series of schemes is to be assured. See ibid., pp. 125-28. 